\title{
Molecular Engineering of Nonplanar Porphyrin and Carbon Nanotube Assemblies: A Linear and Nonlinear Spectroscopic and Modeling Study
}

\author{
Éimhín M. Ní Mhuircheartaigh, ${ }^{1}$ Silvia Giordani, ${ }^{1}$ Donal MacKernan, ${ }^{2}$ Sharon M. King, \\ David Rickard, ${ }^{1}$ Lisa M. Val Verde, ${ }^{1,3}$ Mathias O. Senge, ${ }^{4}$ and Werner J. Blau ${ }^{1}$ \\ ${ }^{1}$ School of Physics, Trinity College Dublin, Dublin 2, Ireland \\ ${ }^{2}$ School of Physics, University College Dublin, Dublin 4, Ireland \\ ${ }^{3}$ Materials Research Laboratory, University of California, Santa Barbara, CA 93106-5121, USA \\ ${ }^{4}$ School of Chemistry, SFI Tetrapyrrole Laboratory, Trinity College Dublin, Dublin 2, Ireland
}

Correspondence should be addressed to Werner J. Blau,wblau@tcd.ie

Received 2 March 2011; Accepted 13 June 2011

Academic Editor: Marisol Reyes-Reyes

Copyright (C) 2011 Éimhín M. Ní Mhuircheartaigh et al. This is an open access article distributed under the Creative Commons Attribution License, which permits unrestricted use, distribution, and reproduction in any medium, provided the original work is properly cited.

\begin{abstract}
The importance of molecular conformation to the nature and strength of noncovalent interactions existing between a series of increasingly nonplanar tetraphenylporphyrin (TPP) derivatives and carbon nanotubes was systematically investigated experimentally in solution using a range of linear and nonlinear optical techniques. Additional complementary molecular dynamics studies were found to support the experimental observations. Convincing evidence of binding between single walled nanotubes (SWNTs) and some of these porphyrins was discovered, and a nonplanar macrocycle conformation was found to increase the likelihood of noncovalent binding onto nanotubes. Nonlinear optical studies showed that the optical limiting behavior of the TPP derivatives deteriorated with increasing porphyrin nonplanarity, but that formation of nanotube composites dramatically improved the optical limiting properties of all molecules studied. It was also found that the significant photoluminescence quenching behavior reported in the literature for such porphyrin/SWNT composites is at least partly caused by photoluminescence and excitation self-absorption and is, therefore, an artifact of the system.
\end{abstract}

\section{Introduction}

Natural cofactors are often found to be tetrapyrroles, due to the high versatility of these organic structures. The functional variety afforded by this class of compounds is related to the flexibility of their molecular conformations, as the physicochemical properties of such molecules may be tuned through the manipulation of the macrocycle. In recent years, porphyrins with such nonplanar macrocyclic conformations have thus attracted considerable attention in an attempt to understand the relationship between conformational distortion and photophysical properties [13]. Single-walled carbon nanotubes (SWNTs) are promising materials for future nanoscale devices, because of their unique structural, mechanical, and electronic properties and have thus attracted much attention since their discovery in
1991 [4]. However, SWNT solubility in aqueous and organic solutions remains a concern and may be achieved chemically while maintaining the superior nanotube electronic structure through noncovalent functionalization [5-9]. Since the initial report by Murakami et al. on nanocomposites formed by van der Waals forces between SWNT and zinc protoporphyrin IX, much research has been conducted on such noncovalent SWNT-porphyrin hybrid materials. Through functionalization with porphyrins, it is hoped that SWNT may gain the superior physicochemical properties of the porphyrin moiety. Indeed, in a previous report by the authors [10], it has been shown that the solubility and optical limiting (OL) properties of carbon nanotubes are enhanced upon interaction with the planar porphyrins tetraphenylporphyrin and protoporphyrin IX in solution [11, 12]. At least, 
two distinct processes are widely believed to contribute to optical limiting of noncovalently bond porphyrin-SWCNT complexes in solution. The first, nonlinear scattering is due to the formation of bubbles in the solvent. This happens at the beginning of a laser pulse, when the leading edge helps heat carbon nanoparticles to form solvent nanobubbles, a process which takes place on a nanosecond time scale. The resulting liquid-gas interface and the associated abrupt change in refractive index lead to intense nonlinear scattering and optical limiting. The second process is considered to consist of reverse saturable absorption (where the absorption cross-section of excited states exceeds that of the ground state) by the porphyrin of the laser, followed by electron, or possibly energy transfer from the excited porphyrin to the SWCNT. Either of these transfer processes is greatly enhanced if the distance between the donor and acceptor is small, as would be the case were the porphyrin to have a tendency to bind noncovalently to the SWCNT. In this scenario, the excited SWCNT relaxes to its ground state nonradiatively through internal electronic-phonon coupling, and interactions with the solvent. Noncovalent interactions between SWNT and nonplanar porphyrins are, therefore, a very interesting novel nanotech approach. Up until now, interactions have been studied between SWNT and predominantly planar porphyrin systems. The correlation, if any, existing between the degree of macrocycle nonplanarity and the strength of the subsequent binding for these nanotube composites is potentially very interesting. Engineering molecules which map onto the nanotube surface appears the logical approach, and, for this reason, a series of six $\beta$-ethylsubstituted tetraphenylporphyrins were synthesized using a technique previously reported $[13,14]$ with varying graded degrees of distortion due to peri-interactions ranging from that of tetraphenylporphyrin $\left(\mathrm{H}_{2} \mathrm{TPP}\right)$ to the standard nonplanar porphyrin 2,3,7,8,12,13,17,18-octaethyl-5,10,15,20tetraphenylporphyrin $\left(\mathrm{H}_{2} \mathrm{OETPP}\right)$ [15]. Porphyrins are also well known for their novel optical nonlinear properties and are, therefore, one of the most studied class of compounds in this field $[2,16,17]$. It has been shown that SWNTs and multiwalled nanotubes (MWNTs) in suspension excited with high intensity at $532 \mathrm{~nm}$ and $1064 \mathrm{~nm}$ also behave as reverse saturable absorbers [18-20]. The interactions between SWNT and the sterically crowded, nonplanar porphyrins investigated in this report in solution are likely to give rise to interesting optical nonlinear behavior for these composite solutions. Thus, the optical properties of carbon nanotubes noncovalently functionalized with nonplanar porphyrin molecules are of enormous interest from the point of view of both their photophysical and nonlinear optical properties and potential applications.

In this paper, we present a comprehensive linear and nonlinear optical characterization of the interaction between the porphyrin and nanotube moieties. We also present evidence that much of the optical evidence attributed to strong noncovalent binding in these systems by other authors is likely to be an artifact, resulting from a well-known optical phenomenon. The experimentally determined linear and nonlinear optical characteristics of these porphyrin composite systems in solution are also compared with the results of a molecular dynamics simulation performed in the NVT ensemble (i.e., the number of particles $N$, the volume $V$, and the temperature $T$ of the system are kept constant), where the tendency exhibited by these tetraphenylporphyrin derivatives to aggregate close to a nanotube surface in the same solvent was investigated.

\section{Experimental Section}

The purified SWNTs used in this work were prepared by the HiPCO process [21], supplied by Carbon Nanotechnologies Inc., and used without further treatment. The nonplanar tetraphenylporphyrin (TPP) derivatives were prepared as described earlier $[13,14]$. The TPP molecules used were purchased from Aldrich. Two identical sets of solutions of each porphyrin were made with concentrations ranging from approximately $1 \mu \mathrm{M}-500 \mu \mathrm{M}$ in dimethylformamide (DMF). Pure HiPCO SWNT was added to one porphyrin solution at each concentration yielding solutions with porphyrinSWNT mass ratios of $1: 1,5: 1$, and $10: 1$.

Photoluminescence measurements were carried out using an LS-55 Perkin-Elmer luminescence spectrometer. The linear absorption spectra were recorded using a Shimadzu UV3100 UV-Vis-NIR spectrometer. Nonlinear optical experiments were performed using the open aperture Z-scan technique [22]. This technique involves scanning the sample through the focus of a Gaussian laser beam, thereby, subjecting it to a large range of intensities. Negligible nonlinear activity occurs initially, when the beam energy density is low, and as the sample approaches the focus, the energy density increases exciting the nonlinear activity within the sample medium. The open aperture Z-scan spectrum acquired during such a scan is the measured beam transmittance as a function of position from the focus, from which the nonlinear extinction coefficient can be extracted. Fitting theory previously reported was used in the calculation of effective absorption coefficients for all systems studied [23]. All experiments were performed using $6 \mathrm{~ns}$ Gaussian pulses from a Q-switched Nd: YAG laser with energies of approximately $0.2-0.3 \mathrm{~mJ}$ per pulse. The beam was spatially filtered to remove the higher-order modes and tightly focused. The laser was operated at its second harmonic, $532 \mathrm{~nm}$, with a pulse repetition rate of $10 \mathrm{~Hz}$. All linear and nonlinear optical measurements were performed in quartz cells of either $1 \mathrm{~cm}$ or $2 \mathrm{~mm}$ path length.

A molecular simulation of the series of nonplanar TPP derivatives in the presence of a 7-7 SWNT in the solvent DMF was also undertaken. All of the simulations were performed using the "COMPASS" force field [24], which is the first force field which has been parameterized and validated using condensed phase properties in addition to various ab initio and empirical data. This molecular force field includes 2, 3, and 4 body terms describing nonbond and bond interactions. The nonbond contributions consist of coulomb (via partial charges) and Van der Waals interactions. The bond (or valence) interactions consist of bond stretching terms, valence angle bending terms, dihedral angle torsion terms, and inversion (also called out-of-plane interactions) terms. 
A 7-7 SWNT has a radius close to that of HiPCO SWNT. This computational work utilized a molecular dynamics simulation (NVT ensemble) to investigate whether the TPP derivatives exhibited enhanced tendencies to aggregate onto the surface of a SWNT in the presence of DMF solvent molecules at standard density $\left(0.944 \mathrm{~g} / \mathrm{cm}^{3}\right)$ and temperature (298 Kelvin). Each system was simulated in a periodic box of size roughly $65 \times 65 \times 41.81 \AA^{3}$, with small differences made to take into account small differences in effective volume of different porphyrin derivatives. The initial conditions in all cases consisted of three layers. The left and right layers each consisted of ten porphyrin molecules in a solvent of $500 \mathrm{DMF}$ molecules, while the middle layer consisted of an uncapped 7-7 SWNT of length $41 \AA$. Each system thus consisted of 20 TPP derivative molecules, 1000 DMF molecules and a single SWNT. Each system was equilibrated for at least $1 \mathrm{ns,}$ and statistics were subsequently collected for a further $0.2 \mathrm{~ns}$. The COMPASS force field was used, and Coulombic and Van der Waals contributions to the forces were computed using a fast: cell multipole technique due to rapid convergence and high precision (almost the same as that obtained with Ewald sums). The NVT ensemble was then sampled using Anderson thermostated molecular dynamics, and an integrating time step of $1 \mathrm{fs}$.

\section{Results and Discussion}

In order to study the correlation between macrocycle nonplanarity for porphyrin molecules and their noncovalent interactions with carbon nanotubes, a series of porphyrins with graded degree of macrocycle distortion was synthesized via mixed condensation of pyrrole, diethylpyrrole, and benzaldehyde as previously described $[13,14]$. The behavior of the nanotube composites formed with these nonplanar porphyrins was compared with that of the more planar molecule tetraphenylporphyrin (TPP).

Limited solubility was observed for the HiPCO SWNT solely in DMF for concentrations below $0.01 \mathrm{gL}^{-1}$. It was found that TPP and its more distorted derivatives 2,3-dieth yl-5,10,15,20-tetraphenylporphyrin (DETPP), 2,3,12,13-tetraethyl-5,10,15,20-tetraphenylporphyrin (tTETPP), 2,3,7, 8-tetraethyl-5,10,15,20-tetraphenylporphyrin (cTETPP), 2, 3,7,8,12,13-hexaethyl-5,10,15,20-tetraphenylporphyrin (HETPP), and 2,3,7,8,12,13,17,18-octaethyl-5,10,15,20-tetraphenylporphyrin (OETPP) could all dissolve SWNT in DMF. Crystallographic studies have previously shown that the macrocycle distortion in the solid state for these molecules increases gradually in the order TPP $<$ DETPP $<$ tTETPP < cTETPP < HETPP < OETPP [13, 14]. Negligible precipitation was noticed in these composite solutions even after several months of storage.

Porphyrin molecules are expected to bind noncovalently to carbon nanotubes through Van der Waals interactions as a result of their high degree of conjugation. Several reports of such noncovalently functionalized carbon nanotube systems exist, including previous studies performed by the authors $[10,25]$. Adhesion of $\mathrm{Zn}$-TPP molecules to the surface of carbon nanotubes has previously been observed with TEM, providing some evidence of interaction between the two moieties in solution. A slow aggregation of the porphyrin: SWNT composite solutions were noticed when the concentration of the dispersion was greater than $59 \mu \mathrm{M}$ for solutions where the porphyrin: nanotube mass ratio was $1: 1$, and approximately $100 \mu \mathrm{M}$ for the $5: 1$ and 10:1 porphyrin: nanotube solutions. This confirmed that noncovalent porphyrin interaction enhanced the solubility of carbon nanotubes in DMF.

The TPP derivative molecules in dimethylformamide all displayed typical absorption spectra, with Soret bands observed ranging between $417 \mathrm{~nm}$ and $453 \mathrm{~nm}$ (Figure 1(a)). The Soret band of each porphyrin was found to shift to a lower energy with increasing porphyrin non-planarity relative to the TPP molecule with bands observed at 417, 420, $424,432,442$, and $453 \mathrm{~nm}$ for TPP, DETPP, tTETPP, cTETPP, HETPP, and OETPP, respectively. The porphyrin nonplanarity results from a gradual distortion of the porphyrin macrocycle due to steric crowding with each additional ethyl group added $[13,14]$. These absorption band red shifts are characteristic of nonplanar porphyrins, where the degree of non-planarity of a molecule is often determined by examination of such spectral shifts $[2,26,27]$.

Upon interaction with the carbon nanotubes, the composite visible-NIR absorption spectra are intermediate between the spectrum of the SWNT suspension and the porphyrin spectrum, with Q bands and Van Hove peaks present, but slightly shifted in some cases (HETPP and OETPP) for the $1: 1$ composite solutions. For the $5: 1$ and $10: 1$ composites, no change in the absorption spectrum was observed, with the composite displaying peaks originating from both the individual porphyrin molecules and the carbon nanotube Van Hove absorption peaks at 377, 409, $451,504,559,602$, and $658 \mathrm{~nm}$. Further evidence of increased nanotube solubility in the presence of the porphyrins was confirmed by the intensity increase of the Van Hove peaks for the composite solutions using UV-visible-near IR absorption spectroscopy (Figure 1(b)).

The fluorescence spectra for excitation at the Soret band of each solution present two emission peaks characteristic of porphyrin photoluminescence for the two most planar samples, TPP and DETPP. The emission deviates from this pattern with increasing macrocycle distortion, with one broad peak observed for tTETPP, cTETPP, and HETPP displaying PL which is barely resolved into two peaks. As the porphyrin macrocycle becomes more perturbed, the luminescence becomes increasingly less intense, with no measurable fluorescence observed for OETPP. The emission wavelengths were also found to red shift with increasing macrocycle nonplanarity $[28,29]$. It was not possible to determine if this was true for the nonfluorescent molecule OETPP. The Stokes shift also increased with increasing molecule nonplanarity, with values of 232, 232, 246, 269, and $308 \mathrm{~nm}$ for TPP, DETPP, tTETPP, cTETPP, and HETPP, respectively. Upon the formation of a $(1: 1)$ composite with the SWNT, the emission profile was unchanged for all porphyrins studied.

In a previous report [10], it was found that both TPP and its metalloporphyrin derivative Zn-TPP displayed 


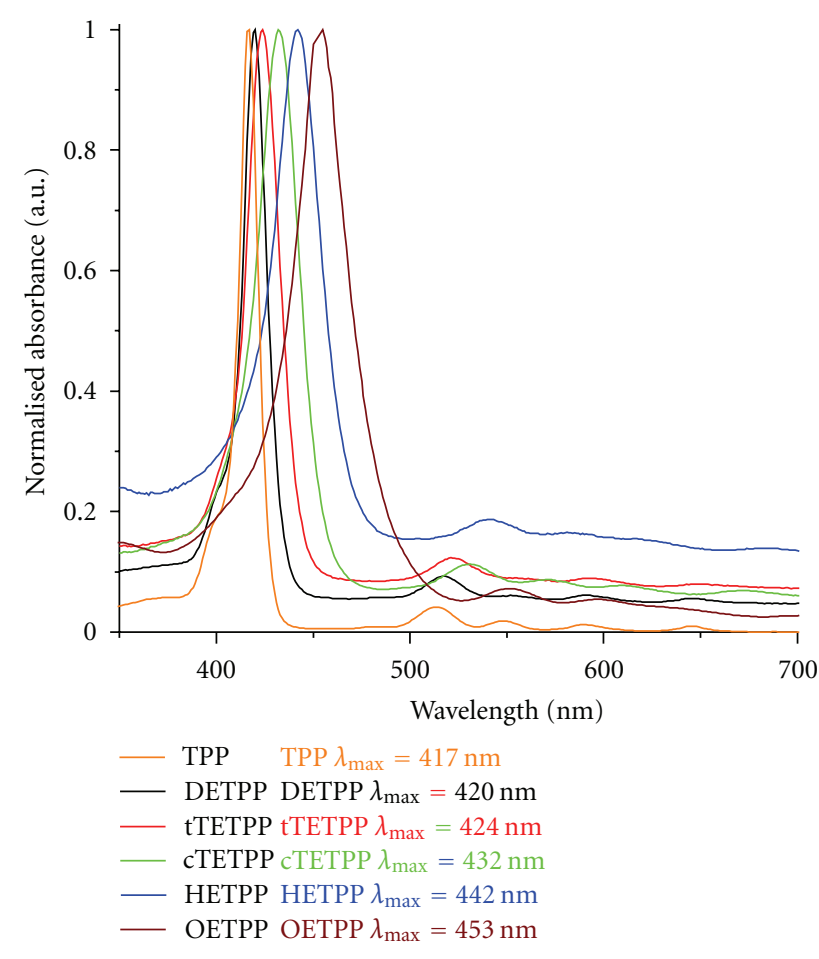

(a)

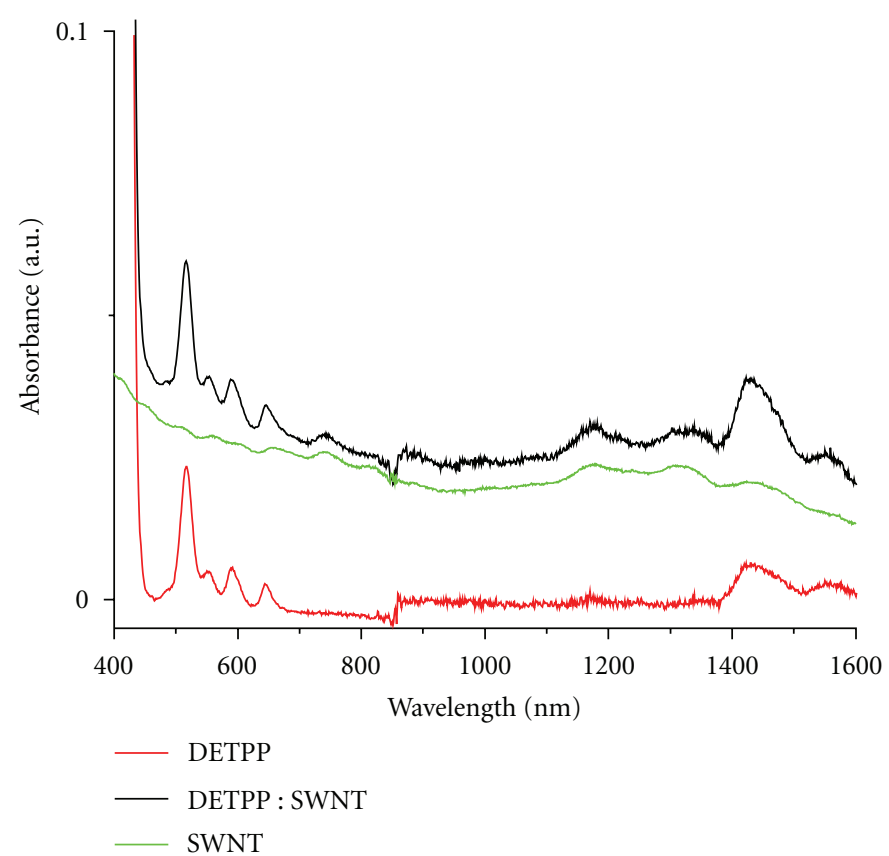

(b)

FIgURE 1: (a) Normalized UV-VIS absorption spectra of TPP, DETPP, tTETPP, cTETPP, HETPP, and OETPP and their (1:1) SWNT composites $\left(0.001 \mathrm{mg} / \mathrm{mL}, \mathrm{DMF}, 25^{\circ} \mathrm{C}\right)$. (b) The VIS-NIR absorption spectra of DETPP, its $(1: 1)$ SWNT composite, and a dispersion of SWNT alone in DMF show the characteristic Q Bands and Van Hove peaks and show that the absorption spectrum of the composite solution is intermediate between that of the porphyrin and the SWNT with characteristics of both.

negligible quenching for $(1: 1)$ composite solutions at porphyrin concentrations below $6.5 \mu \mathrm{M}$, with self-aggregation of the solutions appearing evident at higher concentrations where strong PL quenching was observed for the composite solutions. In the context of this paper, we define aggregation as a generic term which describes any interaction between individual molecules which in turn affects the electronic structure of the individual molecule (including $\pi-\pi$ stacking and other nonpolar interactions). These findings were consistent with the literature, where strong photoluminescence quenching of composite solutions was attributed to evidence of noncovalent binding of porphyrin molecules to carbon nanotubes [30-33]. Such studies were typically performed at only one concentration at the same high concentrations where we had also found strong quenching [10]. In this report, we present evidence that this reported photoluminescence behavior for porphyrin composite solutions is not an effect entailing significant electronic changes in the SWNT's but is largely the result of a common photoluminescence phenomenon. Our findings imply that many of the optical findings which support noncovalent porphyrin functionalization reported in the literature may be artifacts and not actually attributable to either energy transfer or photoinduced electron transfer from the bound porphyrins to the SWNT as suggested. Under commonly used experimental conditions, the observed fluorescence signal from a solution can be found to decrease relative to the sample concentration as the solution becomes increasingly concentrated. This decrease is due, in part, to an attenuation of the excitation beam in regions of the solution in front of the point from which the fluorescence is collected by the detector, and to the absorption of the emitted fluorescence within the solution [34]. This effect is known as the inner filter effect, as defined by Parker and Rees [35].

The variation in photoluminescence intensity was studied as a function of concentration for composite solutions over a wide concentration range. Assuming noncovalent binding of the porphyrins onto the carbon nanotube surface through Van der Waals interactions, the photoluminescence of the composite solutions is expected to be quenched relative to that of the same concentration of porphyrin, consistent with a model developed by Coleman et al. [36] which accurately describes noncovalent nanotube interactions with the conjugated polymer poly [ $m$-phenylenevinylene-co-( $1,5-$ dioctyloxy-2,6-naphthylene vinylene] (pmNV). Due to constant porphyrin adsorption/desorption from the nanotubes, the extent of this photoluminescence quenching is predicted to be highly nonlinear with concentration approaching 1 at very low concentrations.

Concentration-dependent PL studies were performed upon the series of tetraphenylporphyrin derivatives and their $1: 1,5: 1$ and $10: 1$ composites formed with SWNT in order to determine the extent of the noncovalent binding. For the 1:1 DETPP: SWNT solutions, quenching behavior was 


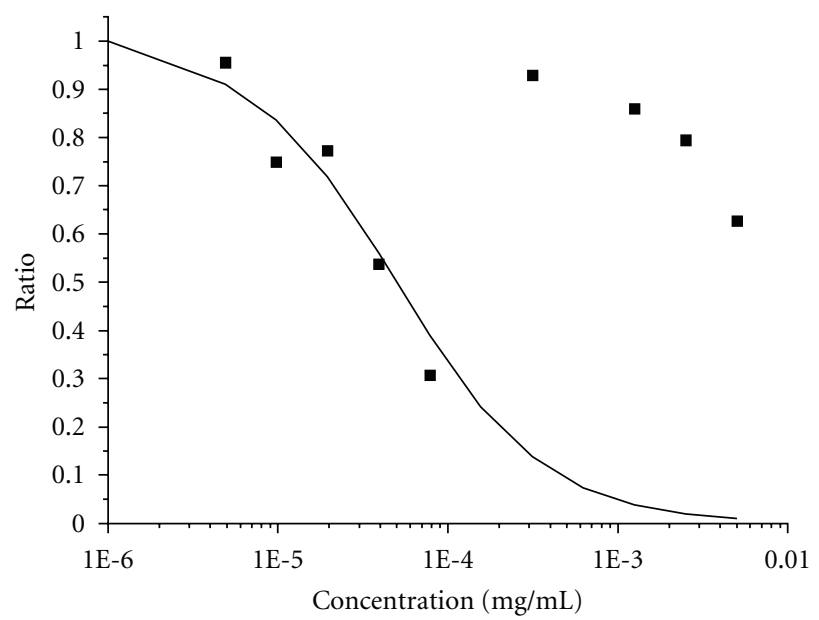

(a)

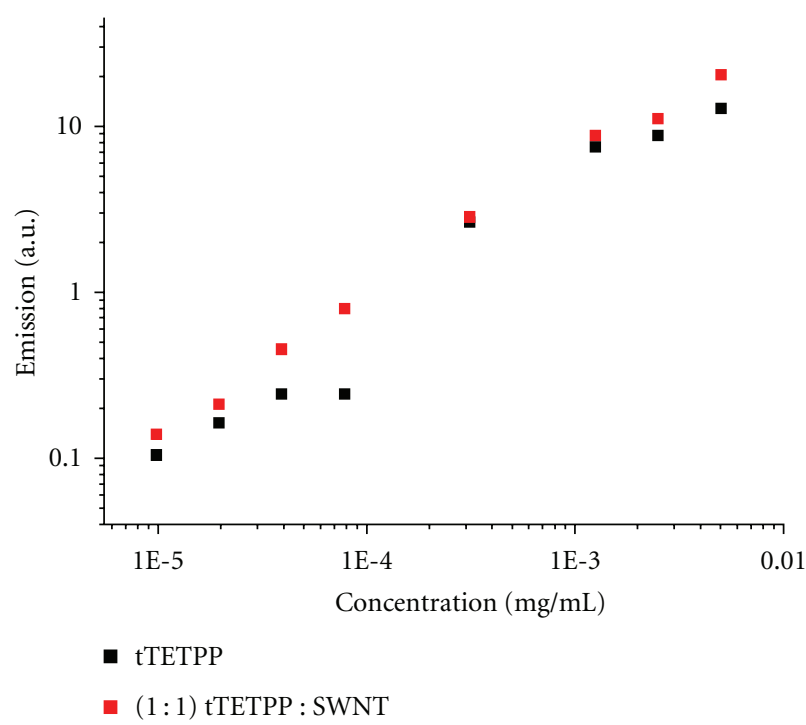

(b)

FIGURE 2: The PL quenching behaviour of tTETPP appeared to be in conservative agreement with the binding model at concentrations sufficiently dilute display an appreciable level of emission quenching.

observed for the composite solutions for concentrations greater than approximately $1 \times 10^{-4} \mathrm{mg} / \mathrm{mL}$ with an appreciable reduction in the porphyrin fluorescence for SWNT composites observed. Our data initially appeared to be in tenuous agreement with the binding model proposed by Coleman et al., with very weak interaction indicated between the nanotubes and the porphyrin solutions. However, this occurred at the onset of the concentration-dependent emission quenching, similar to the behavior previously found for TPP and Zn-TPP. The PL behavior of DETPP displayed only $15 \%$ quenching at sufficiently dilute concentrations where emission intensity was directly proportional to concentration (i.e., for concentrations $<5 \times 10^{-4} \mathrm{mg} / \mathrm{mL}$ ). Negligible quenching was observed in this concentration range for cTETPP and HETPP. It was also observed that both emission bands, particularly the more intense band at $654 \mathrm{~nm}$, were much lower in intensity at higher concentration solutions for spectra normalized at $693 \mathrm{~nm}$. This effect became more marked with increasing concentration, consistent with the inner filter effect. Only $(1: 1)$ composite solutions of one of the porphyrin molecules studied-tTETPP_appeared to display an appreciable level of emission quenching, in conservative agreement with the binding model (Figure 2) at these dilute concentrations. It is worth noting that OETPP was nonfluorescent, and so it was not possible to probe the interaction of this molecule with SWNT using this technique.

Two steps were taken in order to examine the role of the inner filter effect in our studies. Firstly, as one characteristic of the inner filter effect is that fluorescence emission spectra excited in regions of high absorbance are suppressed, the effects on the PL of exciting at different wavelengths were examined. Secondly, it is well documented that cell geometry is of great importance in the inner filter effect. To minimize this phenomenon at high concentrations, the $1 \mathrm{~cm}$ quartz cuvette was replaced with a $2 \mathrm{~mm}$ quartz cuvette, which was mounted in a specially designed holder within the fluorimeter. The influence of the inner filter effect was immediately apparent upon examination of composite solutions containing 10:1 porphyrin: SWNT when both steps were taken to minimize this effect. Additionally, the solutions were all photoexcited at a number of wavelengths, namely, in the Soret and Q-bands of the porphyrin, and in a region of no absorbance. The difference in the emission for higher concentration solutions was significant. Concentration-induced self-absorption was greatly reduced by altering the excitation wavelength. It is apparent that the inner filter effect is responsible for much of the PL quenching observed for composite solutions at high concentration, as this effect was greatly enhanced in the presence of SWNT. This is probably due to the combination of light being scattered by the nanotubes in addition to the self-absorption of the porphyrin molecules present. Hence, strong apparent composite PL quenching was observed for all molecules studied when appropriate precautions were not taken to counter the effects of the inner filter effect.

Taking this into account, PL studies of all molecules were conducted. Plots of TPP and its 10:1 composite emission upon photoexcitation in the Soret band region at $417 \mathrm{~nm}$ (top) and the Q band region at $590 \mathrm{~nm}$ (bottom) for TPP as a function of concentration for high concentration solutions are shown in Figure 3. Also shown is the ratio of composite to porphyrin emission as a function of concentration upon excitation at both wavelengths. The $2 \mathrm{~mm}$ cuvette was used for all solutions at concentrations higher than $0.001 \mathrm{mg} / \mathrm{mL}$. It is evident that negligible quenching is found for the composite solutions upon excitation at both wavelengths. This is in direct contrast to our previous studies with 1:1 TPP : SWNT composites, where 92\% "quenching" was observed for composites at a concentration of $0.036 \mathrm{mg} / \mathrm{mL}$. As this result was consistent with those reported in literature, 


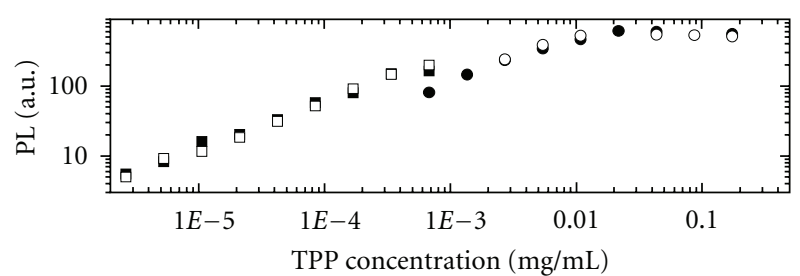

(a)

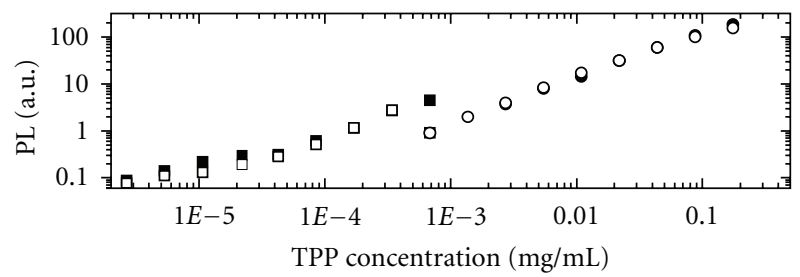

(b)

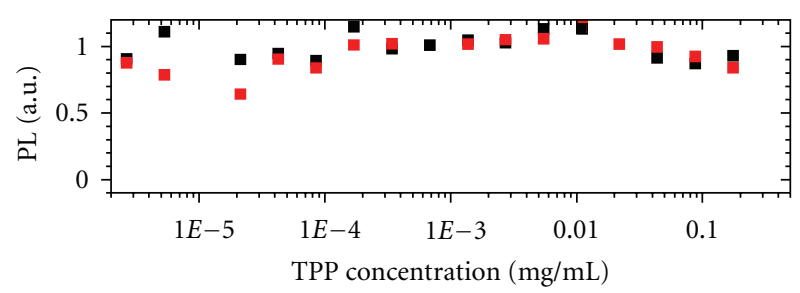

(c)

FIgURe 3: PL emission of TPP and its 10: 1 SWNT composite upon photoexcitation in the Soret band region at $417 \mathrm{~nm}$ (a) and the Q band region at $590 \mathrm{~nm}$ (b) as a function of concentration for high concentration solutions. Also shown is the ratio of composite to porphyrin emission as a function of concentration upon excitation at both wavelengths. The 2-mm cuvette was used for all solutions at concentrations higher than $0.001 \mathrm{mg} / \mathrm{mL}$.

it is likely that many other studies have failed to correct for the inner filter effect.

Some quenching was in fact found for the $10: 1$ tTETPP composites. However, the quenching remained approximately constant over the concentration range $0.001-$ $0.175 \mathrm{mg} / \mathrm{mL}$, yielding a median quenching of $25.2 \%$. No quenching whatsoever was evident for $(10: 1)$ solutions of cTETPP, HETPP, and DETPP. For comparison purposes, $(10: 1)$ solutions of the planar molecule octaethylporphyrin (OEP) were also studied, and no change was noted in the PL intensity levels upon the addition of nanotubes, except that the luminescence was possibly slightly enhanced.

A series of $(5: 1)$ porphyrin: SWNT solutions were also examined for the planar molecules TPP and OEP. In both cases, the composite luminescence appeared to be enhanced relative to that of the same concentration of porphyrin. As the interaction of the porphyrin molecules with nanotubes is expected to quench the emission intensity, if anything, it is clear that the system is not as straightforward as initially thought. One possible explanation for this behavior is that the porphyrin molecules aggregate in DMF, and that interaction with the nanotubes prevents this aggregation from occurring. Although these molecules do not bind to the nanotube surface in a way which facilitates photoinduced electron transfer, the noncovalent interactions between them appear stronger than the attractive forces between adjacent porphyrin molecules. Interestingly, (1:1) Zn-TPP: SWNT solutions also exhibited composite luminescence which was approximately $17 \%$ higher than those of the porphyrin solutions for concentrations lower than $1.7 \times 10^{-4} \mathrm{mg} / \mathrm{mL}$. Our results for $(1: 1)$ TPP: SWNT were inconclusive at these concentrations.

The absence of PL quenching observed for the $(10: 1)$ composite solutions of these molecules is also consistent with this theory. With a greater mass of porphyrins than nanotubes present in these solutions by an order of magnitude, it is clear that this quantity of nanotubes has no measurable impact on the degree of self-aggregation. With fewer SWNT present in solution, fewer sites for porphyrin aggregation are provided, and consequently the SWNTs have less effect on the porphyrins present in solution. The ratio of composite to porphyrin PL as a function of concentration is shown below for the 5:1 TPP : SWNT solutions, showing that the composite luminescence is higher than that of its corresponding TPP solution over the concentration range studied. These results are consistent with the molecular simulation results where a pronounced tendency to aggregate both onto the SWNT surface and onto each other was exhibited by these porphyrin molecules (Figure 4).

Increasing the ratio of porphyrin to SWNT in solution from $1: 1$ (to $5: 1$ and $10: 1$ ) porphyrins to nanotube by weight, it was found that maximum porphyrin concentrations of $0.04375 \mathrm{mg} / \mathrm{mL}$ and $0.175 \mathrm{mg} / \mathrm{mL}$ were achieved for the $5: 1$ and 10: 1 composites, respectively, before the SWNT aggregated out of solution, equivalent to $0.00875 \mathrm{mg} / \mathrm{mL}$ and $0.0175 \mathrm{mg} / \mathrm{mL}$ of SWNT, respectively. As a maximum SWNT concentration of approximately $0.01 \mathrm{mg} / \mathrm{mL}$ was achieved for the $1: 1$ composite solutions, the maximum nanotube concentration appears to remain approximately constant, irrespective of the number of porphyrin molecules available to aid solubilization.

3.1. Nonlinear Optical Results. The open aperture of a Zscan experiment was used to measure the total transmittance through the samples [37]. Effective absorption coefficients were calculated by fitting theory previously reported [23]. Normalized transmittance as a function of position $z, T_{\text {Norm }}(z)$ was given by

$$
T_{\text {Norm }}(z)=\frac{\ln \left[1+q_{0}(z)\right]}{q_{0}(z)},
$$

where $q_{0}(z)=q_{00} /\left(1+\left(z / z_{0}\right)^{2}\right), z_{0}$ is the diffraction length of the beam, $q_{00}=\beta_{\text {eff }} I_{0} L_{\text {eff. }}$.

Here, $L_{\text {eff }}=\left[1-\exp \left(-\alpha_{0} L\right)\right] / \alpha_{0}, \beta_{\text {eff }}$ is the effective intensity-dependent nonlinear absorption coefficient, and $I_{0}$ is the intensity of the light at focus. $L_{\text {eff }}$ is known as the effective length of the sample defined in terms of the linear absorption coefficient, $\alpha_{0}$, and the true optical path length through the sample, $L$. The imaginary third-order optical susceptibility $\operatorname{Im}\left\{\chi^{(3)}\right\}$ is directly related to the intensitydependent absorption coefficient $\beta_{I}$ and is expressed as 


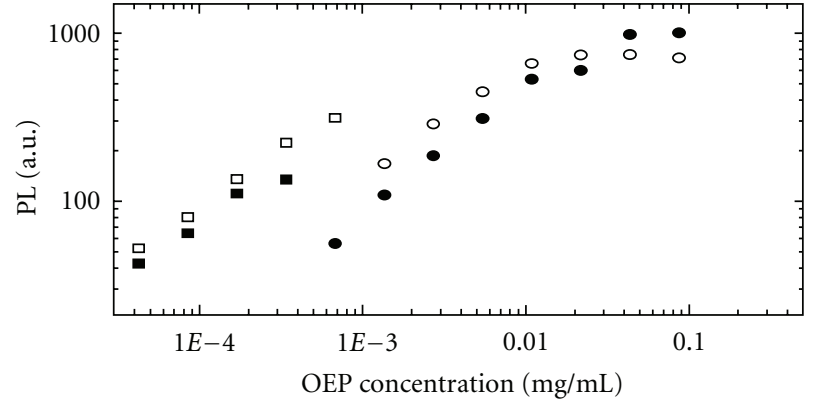

(a)

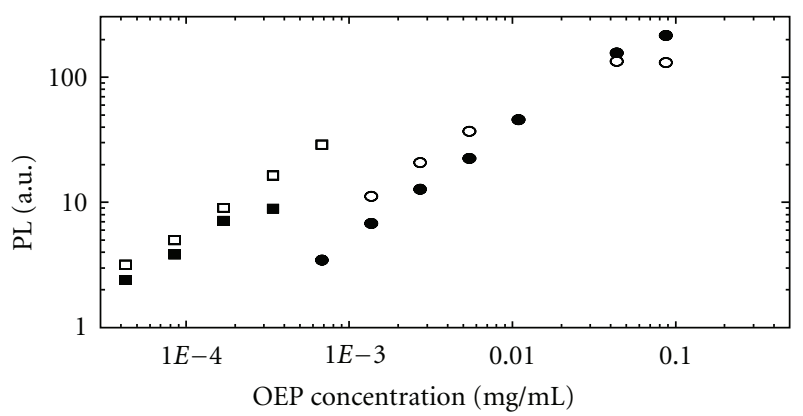

(c)

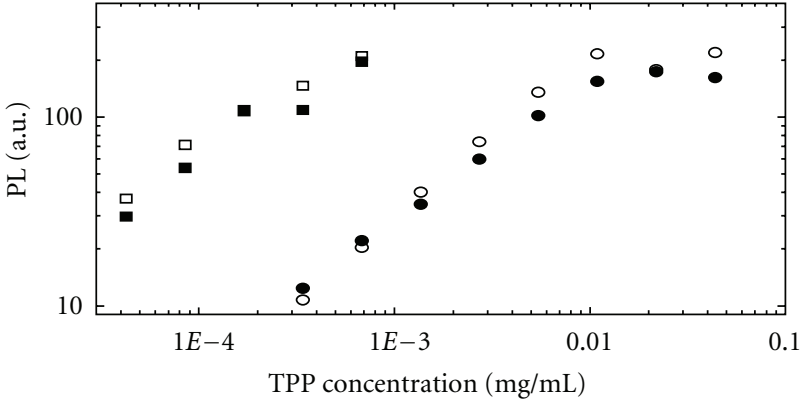

(b)

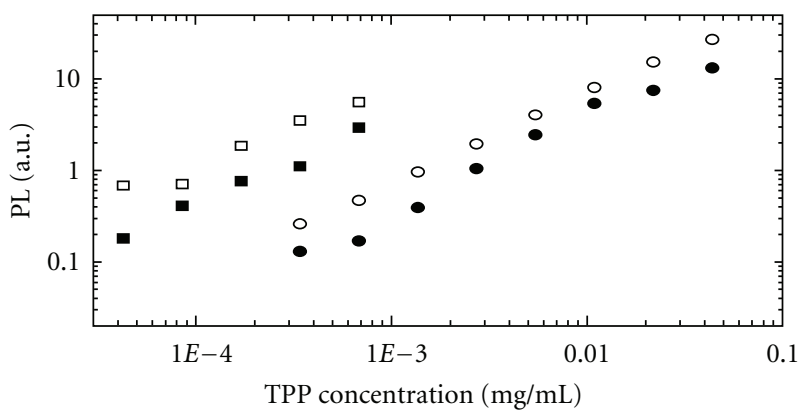

(d)

FIGURE 4: PL intensity as a function of concentration for 5:1 porphyrin: SWNT solutions for OEP (a, c) and TPP (b, d) show enhanced luminescence for composites. These solutions were photoexcited at the Soret band (a, b) (396 nm and $417 \mathrm{~nm}$ for OEP and TPP, resp.) and Q band (c, d) $(529 \mathrm{~nm}$ and $590 \mathrm{~nm}$ for OEP and TPP, resp.) wavelengths. Measurements were performed in $2 \mathrm{~mm}$ quartz cuvettes for concentrations higher than $0.0003 \mathrm{mg} / \mathrm{mL}$. A $1 \mathrm{~cm}$ quartz cuvette was used for all other measurements.

$\operatorname{Im}\left\{\chi^{(3)}\right\}=n_{0}^{2} \varepsilon_{0} c \lambda \beta_{I} / 2 \pi$, where $n_{0}$ is the linear refractive index, $\varepsilon_{0}$ is the permittivity of free space, $c$ is the speed of light, and $\lambda$ is the wavelength of the incident light. All experiments described in this study were performed using $6 \mathrm{~ns}$ Gaussian pulses from a Q-switched Nd: YAG laser with energies of approximately $0.2-0.3 \mathrm{~mJ}$ per pulse. A normalized energy transmission without an aperture (i.e., open aperture) is shown in Figure 5 for a range of input energies at low onfocus intensities. All tetraphenylporphyrin derivatives were studied at a concentration of $0.01 \mathrm{mg} / \mathrm{mL}$ along with their single-wall nanotube composite solutions, with linear transmission in the region of $50-80 \%$. These solutions were prepared and studied in DMF.

All Z scans performed in this study exhibited a reduction in transmittance about the focus at low intensities (see Figure 5). This is typical of nonlinear absorption of incident light being induced in the sample. For higher incident intensities $\left(I_{0}>\sim 0.2 \mathrm{GW} / \mathrm{cm}^{2}\right)$, all of the nonplanar porphyrins were found to completely decompose the transmission of the porphyrin solutions about the focus displaying apparent saturable absorption (SA). Confirmation that this was a degradation effect as opposed to genuine SA taking place came from examination of the Z-scan traces obtained. Similar degradation effects have been seen in recent studies of optical limiting effects in linear carbon chains in water [38].

For an open aperture scan, the transmission as a function of distance from the focus typically changes symmetrically. More importantly, the initial and final transmission should be the same for a given scan (i.e., $100 \%$ after normalization of these plots). It can clearly be seen that this is not the case for the nonplanar porphyrin scans, an example of which is shown in Figure 5(f). For each case, the transmittance after the scan is much higher than the initial transmittance, indicating the destruction of materials present in the solution.

All composite Z scans performed in this study exhibited a reduction in transmittance about the focus. This is typical of nonlinear absorption of incident light being induced in the sample. As previously [10] reported for TPP composites, introduction of carbon nanotubes into the system completely prevented this degradation at all irradiances, most likely due to the remarkable thermal conduction properties of carbon nanotubes preventing local heating in these solutions.

The nonlinear absorption coefficient, $\beta_{I}$, experienced by the incident pulses was determined from these spectra as a function of the onfocus intensity. The values of $\beta_{I}$ and $\operatorname{Im}\left\{\chi^{(3)}{ }_{\text {eff }}\right\}$ were found to decrease in magnitude as nonplanarity increased. This means that deteriorating optical limiting behavior is observed as molecules become more nonplanar. No trend was observed in the value of $\operatorname{Im}\left\{\chi^{(3)}\right\}$ as a function of planarity for the composite solutions. The composites were found to be better optical limiters than porphyrin molecules alone. All nonplanar porphyrins were found to be inferior optical limiters to TPP, SWNTs alone were found to be superior optical limiters to all porphyrins studied except TPP and DETPP, and inferior to all composite solutions. 


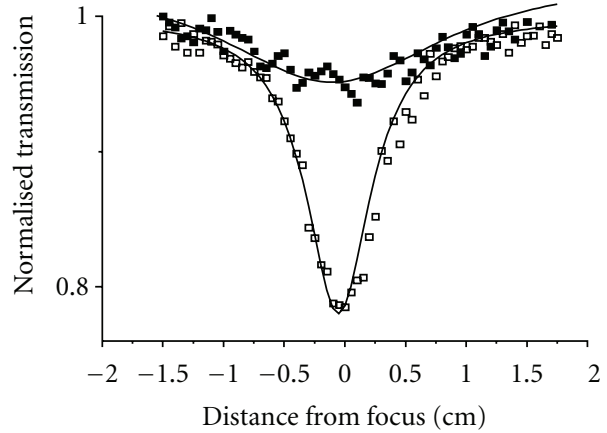

(a)

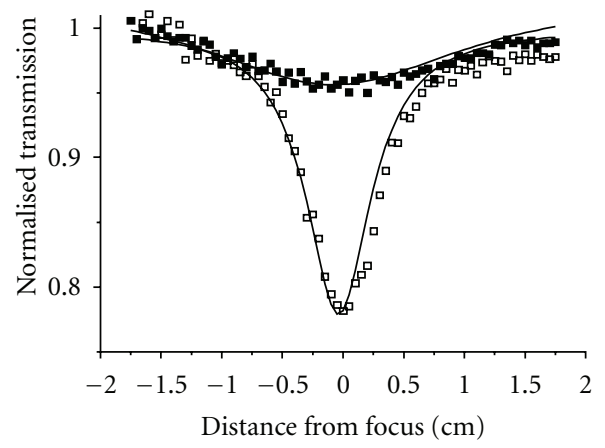

(c)

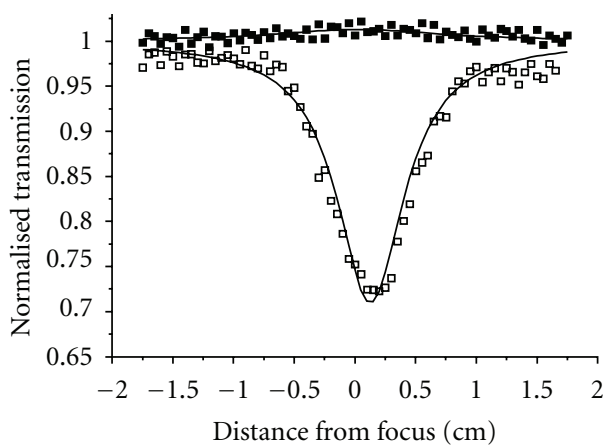

(e)

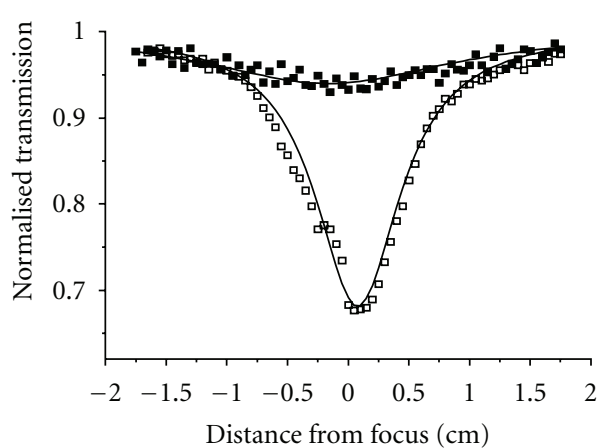

(b)

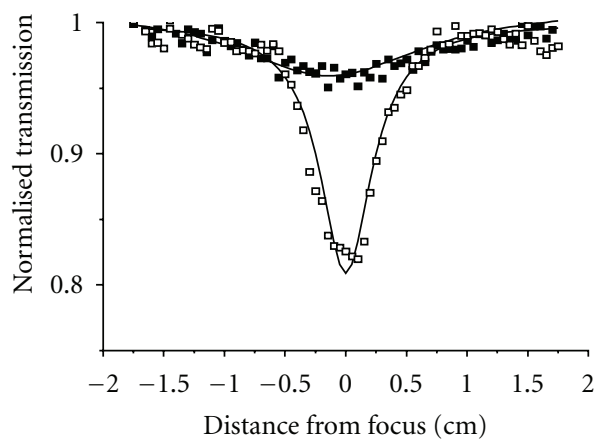

(d)

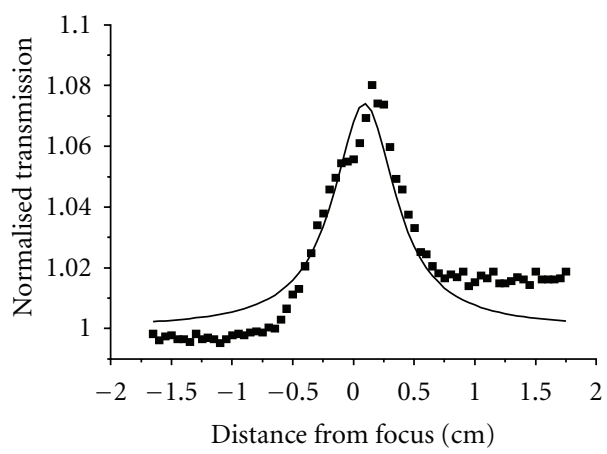

(f)

FIGURE 5: Plots of normalised transmission versus distance from focus for porphyrin and composite solutions at low energies for (a) DETPP, (b) tTETPP, (c) cTETPP, (d) HETPP, and (e) OETPP. (f) The higher final transmittance compared with the initial transmittance indicates the destruction of molecules present in the solution. Porphyrin and composite solutions were at concentrations of $0.01 \mathrm{mg} / \mathrm{mL}$, with onfocus intensities of (a) 0.12 , (b) 0.13 , (c) 0.14 , (d) 0.25 , (e) 0.23 , and (f) $1.63575 \mathrm{GW} / \mathrm{cm}^{2}$ for porphyrin solutions and (a) 0.25 , (b) 0.16 , (c) 0.24 , (d) 0.23 , and (e) $0.19 \mathrm{GW} / \mathrm{cm}^{2}$ for composite solutions.

In the case of three of the nonplanar molecules- tTETPP, HETPP, and OETPP-the optical limiting characteristics of the composites were found to be superior to the combination of the composite components. This was determined by comparing the product of the $\%$ transmittance of the constituent porphyrin and SWNT with the \% transmittance of the composite at the same concentration for a range of energies. If the superior OL behavior of a composite was simply due to an addition of its two optical limiting components, then the transmission shown by the composite should be equal to the product of the transmittance spectra of its porphyrin and nanotube parts. This was found to be the case for DETPP and cTETPP, indicating negligible interaction between these porphyrins and the carbon nanotubes in solution.
The optical limiting curves are shown in Figure 6. Normalized transmission $\left(T_{\text {norm }}\right)$ was plotted as a function of incident pulse energy density $\left(\mathrm{J} \mathrm{cm}^{-2}\right)$. The nonlinear absorption coefficient $\alpha\left(F, F_{\text {sat }}, \kappa\right)$ was used to fit the normalized transmission as a function of this energy density to the data sets of all open aperture $\mathrm{Z}$ scans performed for each compound over a concentration range. Here, $\alpha\left(F, F_{\text {sat }}, \kappa\right)$ is derived from laser rate equations for the steady state and is defined as $\alpha\left(F, F_{\text {sat }}, \kappa\right) \approx \alpha_{0}\left(1+F / F_{\text {sat }}\right)^{-1}\left(1+\kappa F / F_{\text {sat }}\right)$, where $F$ represents the energy density, $F_{\text {sat }}$ the saturation energy density, and $\kappa$ the ratio of the excited to ground state absorption cross-sections, as previously described [10]. The optical limiting (OL) curves (plots of normalized transmission against pulse energy density) for all solutions 


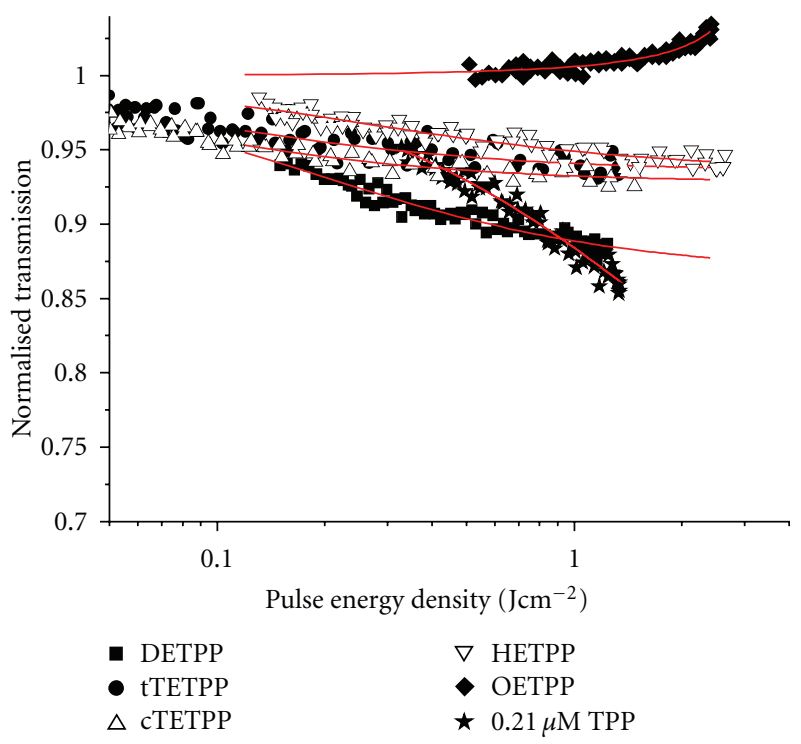

(a)

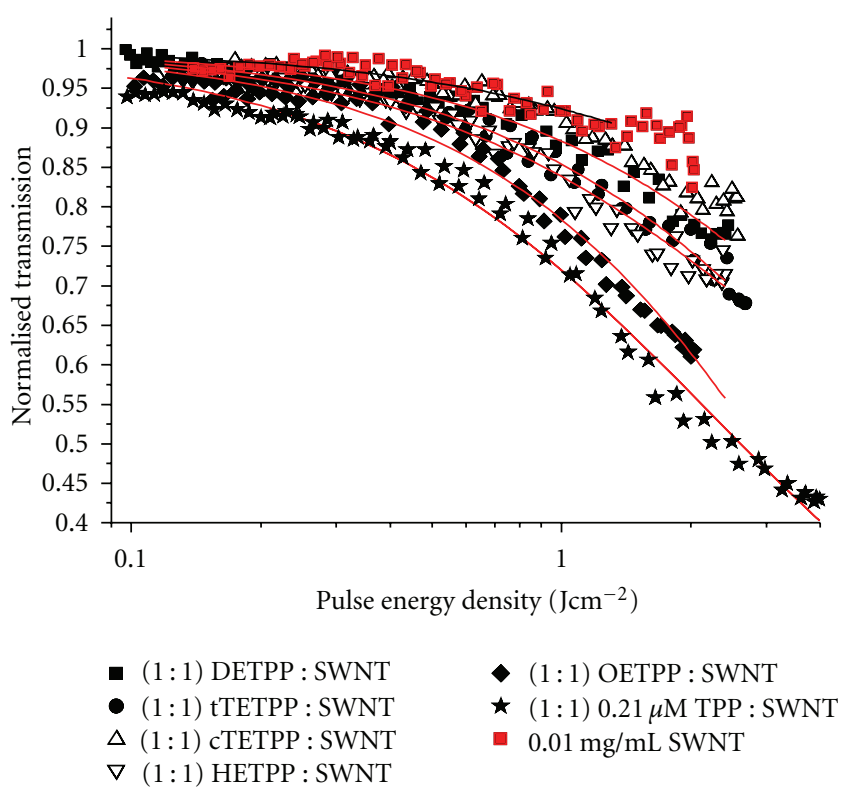

(b)

FIGURE 6: Superior optical limiting behaviour of porphyrin composites is clearly to be seen in these plots of normalised transmission versus pulse energy density. All plots are for onfocus beam intensity $I_{0}$ of $0.2 \mathrm{GW} / \mathrm{cm}^{2}$ (approximately).

TABLE 1: Optical coefficients for the materials measured.

\begin{tabular}{|c|c|c|c|c|c|c|c|c|}
\hline Sample & $\begin{array}{c}c \\
\mu \mathrm{M}\end{array}$ & Solvent & $\begin{array}{c}\alpha_{0} \\
\mathrm{~cm}^{-1}\end{array}$ & $\begin{array}{c}\beta_{I} \\
\mathrm{Cm} \mathrm{W}^{-1}\end{array}$ & $\begin{array}{c}\operatorname{Im}\left\{\chi^{(3)}{ }_{\text {eff }}\right\} \\
\text { Esu }\end{array}$ & $\begin{array}{c}I_{0} \\
\mathrm{GW} \mathrm{cm} \mathrm{cm}^{-2}\end{array}$ & $\begin{array}{c}F_{\text {sat }} \\
\mathrm{J} \mathrm{cm}^{-2}\end{array}$ & $\begin{array}{c}\kappa \\
\sigma_{\mathrm{ex}} / \sigma_{0}\end{array}$ \\
\hline TPP & 21.5 & DMF & 0.11 & $2.84 \times 10^{-9}$ & $9.74 \times 10^{-13}$ & 0.13 & $2.14 \pm 0.22$ & $4.38 \pm 0.24$ \\
\hline $\mathrm{TPP}+\mathrm{SWNT}$ & 21.5 & DMF & 0.42 & $6.22 \times 10^{-9}$ & $2.14 \times 10^{-12}$ & 0.19 & $4.14 \pm 0.31$ & $3.62 \pm 0.15$ \\
\hline DETPP & $0.01 \mathrm{~g} / L=c .16 \mu \mathrm{M}$ & $\mathrm{DMF}$ & 0.25 & $2.63 \times 10^{-9}$ & $9.03 \times 10^{-13}$ & 0.12 & $0.20 \pm 0.01$ & $1.57 \pm 0.01$ \\
\hline DETPP + SWNT & c. $16 \mu \mathrm{M}$ & DMF & 0.55 & $5.49 \times 10^{-9}$ & $1.89 \times 10^{-12}$ & 0.25 & $17.86 \pm 7.02$ & $5.30 \pm 1.54$ \\
\hline tTETPP & c. $16 \mu \mathrm{M}$ & DMF & 0.24 & $1.17 \times 10^{-9}$ & $4.03 \times 10^{-13}$ & 0.13 & $0.09 \pm 0.01$ & $1.28 \pm 0.01$ \\
\hline tTETPP + SWNT & c. $16 \mu \mathrm{M}$ & DMF & 0.57 & $1.06 \times 10^{-8}$ & $3.65 \times 10^{-12}$ & 0.16 & $14.30 \pm 3.42$ & $5.27 \pm 0.90$ \\
\hline сTETPP & c. $16 \mu \mathrm{M}$ & DMF & 0.42 & $1.33 \times 10^{-9}$ & $4.57 \times 10^{-13}$ & 0.14 & $0.07 \pm 0.004$ & $1.18 \pm 0.003$ \\
\hline cTETPP + SWNT & c. $16 \mu \mathrm{M}$ & DMF & 0.69 & $3.35 \times 10^{-9}$ & $1.15 \times 10^{-12}$ & 0.24 & $18.42 \pm 8.60$ & $3.81 \pm 1.19$ \\
\hline HETPP & c. $16 \mu \mathrm{M}$ & DMF & 0.20 & $5.6 \times 10^{-10}$ & $1.92 \times 10^{-13}$ & 0.25 & $0.26 \pm 0.02$ & $1.33 \pm 0.01$ \\
\hline HETPP + SWNT & c. $16 \mu \mathrm{M}$ & DMF & 0.65 & $5.84 \times 10^{-9}$ & $2.01 \times 10^{-12}$ & 0.23 & $6.74 \pm 1.20$ & $3.11 \pm 0.30$ \\
\hline OETPP & c. $16 \mu \mathrm{M}$ & DMF & 0.20 & $-2.82 \times 10^{-10}$ & $-9.68 \times 10^{-14}$ & 0.23 & $-3.79 \pm 0.21$ & $1.08 \pm 0.01$ \\
\hline OETPP + SWNT & c. $16 \mu \mathrm{M}$ & DMF & 0.73 & $1.18 \times 10^{-8}$ & $4.07 \times 10^{-12}$ & 0.19 & $\mathrm{n} / \mathrm{a}^{*}$ & $\mathrm{n} / \mathrm{a}^{*}$ \\
\hline SWNT & $0.01 \mathrm{~g} / \mathrm{L}$ & DMF & 0.29 & $1.87 \times 10^{-9}$ & $6.41 \times 10^{-13}$ & 0.19 & $5.28 \pm 2.18$ & $2.72 \pm 0.56$ \\
\hline
\end{tabular}

* These data did not conform to the model, probably because the optical nonlinearities were predominantly caused by scattering in this composite system, whereas the fit assumes purely absorption-based optical nonlinearities.

are shown in Figure 6 where the solid lines are theoretical fits to the experimental data. All values of $\alpha_{0}, \kappa$, and $F_{\text {sat }}$ calculated for each compound are presented in Table 1 . Reported values calculate an absorption cross-section $\kappa$ of $(30 \pm 6)$ and $\beta_{I}$ of approximately $4.5 \times 10^{-8} \mathrm{~cm} / \mathrm{W}$ at an irradiation of $0.4 \mathrm{GW} / \mathrm{cm}^{2}$ for the benchmark molecule chloro (phthalocyaninato) indium [39].

3.2. Computational Results. It was found through molecular simulation of these porphyrin composite systems that some porphyrin molecules DETPP and tTETPP in particular did exhibit a pronounced tendency to aggregate onto the SWNT surface, or at closest remove within 4-5 angstroms of the SWNT with no DMF solvation layer present. However, other porphyrins-TPP and CTETPP_in particular exhibited no tendency to aggregate onto the SWNT in these studies. All porphyrin molecules studied exhibited a tendency to selfaggregate. These observations are summarized in Table 2 below, where $N$ denotes the number of porphyrin molecules in contact with the SWNT, and $M$ denotes the largest porphyrin derivative self-aggregate. The observations were made over .2 ns interval subsequent to the 1 ns equilibration period, by which time the values of $N$ and $M \operatorname{did}$ not fluctuate appreciably. 


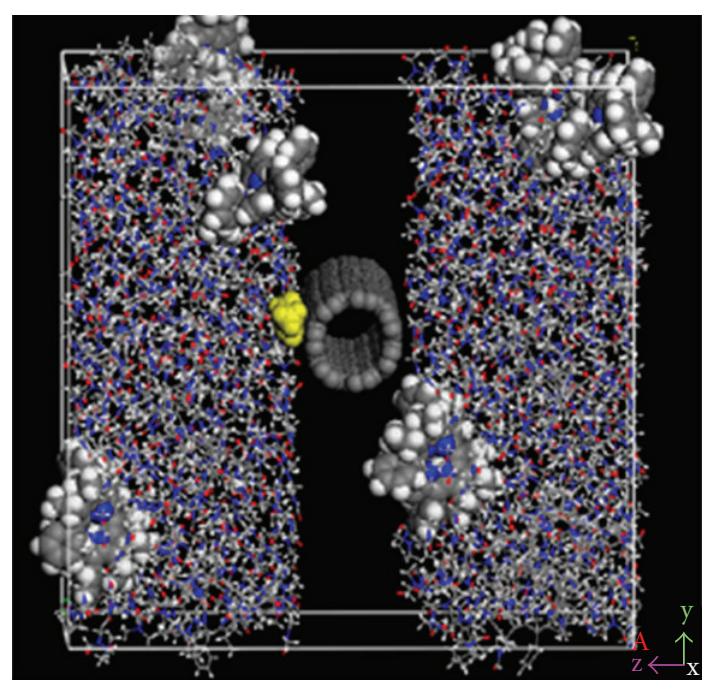

(a)

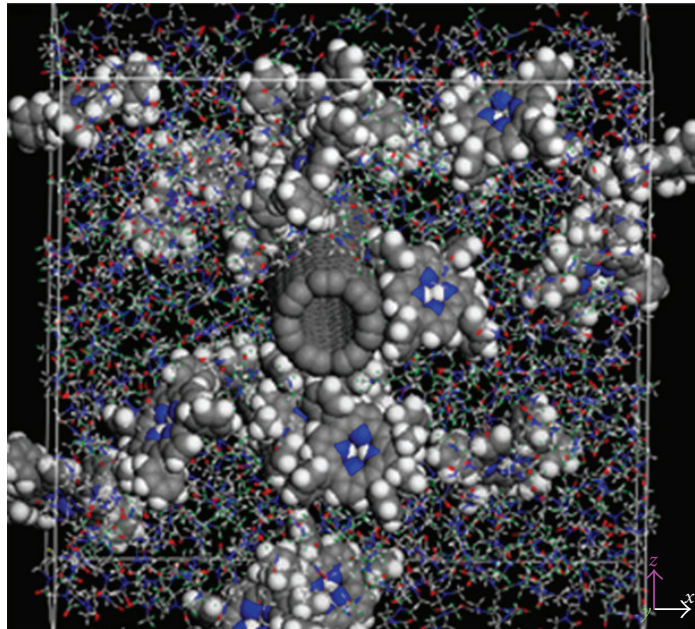

(b)

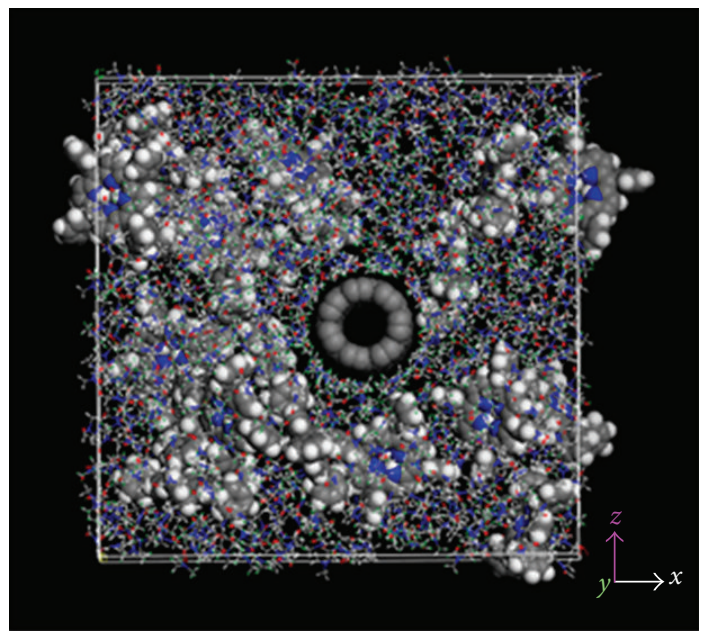

(c)

Figure 7: (a) Simulation initial conditions are shown for the HETPP-SWNT-DMF system. While there are 20 HETPP molecules, only 4 HETPP molecules, a single DMF (yellow) molecule, and the SWNT are actually rendered using effective Van der Waals diameters. The box is in fact repeated in all directions periodically, to simulate a bulk solvent, and an infinitely long SWNT. (b) The DETPP-SWNT-DMF solvent system. The pronounced tendencies of DETPP to aggregate on, and close to the SWNT, are evident. (c) The TPP-CNT-DMF solvent system. There is no tendency for TPP to aggregate on or close to the CNT.

TABLE 2: Summary of porphyrin aggregation behaviour.

\begin{tabular}{lcc}
\hline Sample & $N$ & $M$ \\
\hline TPP & 0 & 3 \\
DETPP & 4 & 5 \\
tTETPP & 4 & 3 \\
cTETPP & 0 & 3 \\
HETPP & 3 & 3 \\
OETPP & 3 & 5 \\
\hline
\end{tabular}

$N$ denotes the number of porphyrin molecules in contact with the SWNT, and $M$ denotes the largest porphyrin derivative self-aggregate.

The two extreme cases, DETPP and TPP, are presented in Figure 7 below, along with the starting conditions for the simulations. The simulations revealed the tendency of certain porphyrin molecules to have enhanced aggregation abilities onto the CNT, roughly in line with experiment. All porphyrin derivatives additionally exhibited a tendency to self-aggregate. This tendency was confirmed by running simulations for the porphyrin molecules alone both in pure DMF solvent and in vacuum in the absence of SWNT.

\section{Conclusion}

In conclusion, linear and nonlinear optical techniques have been of value in clarifying the existence and extent of noncovalent interaction between a series of aromatic tetraphenylporphyrin derivatives and carbon nanotubes in solution. This series of TPP derivatives provided a means of systematically investigating the importance of porphyrin 
macrocycle conformation on the strength of this noncovalent interaction between these two moieties. Convincing evidence of binding between SWNT and three of these porphyrins was discovered using a number of experimental techniques. Absorption spectroscopy indicated binding of HETPP and OETPP, the two most non-planar porphyrins studied, onto the surface of the SWNT in solution. The evidence for their binding came from shifts in the porphyrin Q bands and some of the carbon nanotube Van Hove peaks in the absorption spectra measured for these systems. The tTETPP molecule did not show these shifts in the absorption spectra but was found to exhibit photoluminescence quenching for the composite solutions. The PL quenching followed by this system was found to be highly nonlinear when plotted as a function of nanotube concentration, and in excellent agreement with a theoretical model which has been found to accurately describe the noncovalent binding of conjugated organic moieties onto the surfaces of SWNT in solution. The tTETPP molecule was the only porphyrin found to obey this binding model. DETPP also displayed weak $(\sim 15 \%)$ PL quenching for dilute $1: 1$ composite solutions. It was not possible to assess the interaction of the most nonplanar molecule OETPP with SWNT using these concentrationdependent PL studies, as this molecule did not emit any measurable fluorescence. Nonlinear optical studies using the Z-scan technique showed that the nonlinear absorption coefficient, and, therefore, the optical limiting behavior, of the TPP derivatives studied deteriorated with increasing porphyrin nonplanarity. It was found that the formation of nanotube composites dramatically improved the optical limiting properties of all molecules studied. Evidence of interaction between the SWNT and the porphyrin molecules was found for tTETPP, HETPP, and OETPP from their optical limiting performance, consistent with the results found from the linear optical studies. No evidence of interaction was found experimentally for the molecules TPP and CTETPP. In general, while it was found that the more nonplanar porphyrin molecules have a higher likelihood of noncovalent binding onto nanotubes, it is clear that this is not the only factor determining the noncovalent interaction between them. It was also found that the significant photoluminescence quenching behavior reported in literature for porphyrin composite solutions, and subsequently attributed to electron or energy transfer from the porphyrin molecule to the nanotube, is at least partly caused by photoluminescence and excitation self absorption. Complementary molecular dynamics studies were in striking agreement with these experimental observations. It should be noted that DMF is a very polar solvent, and other solvents may exhibit different properties. Therefore, it may also be of interest to examine other solvents and their effects on these systems.

\section{Acknowledgments}

This work was supported by research Grants from Science Foundation Ireland SFI P.I. 09/IN.1/B2650 (for M. O. Senge), SFI/05RFP/CHE0100 (for W.J.Blau), and by SFI 08-IN.1I1869 (for D.Mackernan).

\section{References}

[1] M. O. Senge, "The conformational flexibility of tetrapyrroles-current model studies and photobiological relevance," Journal of Photochemistry and Photobiology. B, vol. 16, no. 1, pp. 3-36, 1992.

[2] M. O. Senge, "Exercises in molecular gymnastics-bending, stretching and twisting porphyrins," Chemical Communications, no. 3, pp. 243-256, 2006.

[3] M. Ravikanth and T. K. Chandrashekar, "Nonplanar porphyrins and their biological relevance: ground and excited state dynamics," Structure and Bonding, vol. 82, pp. 105-188, 1995.

[4] S. Iijima, "Helical microtubules of graphitic carbon," Nature, vol. 354, no. 6348, pp. 56-58, 1991.

[5] H. Murakami, T. Nomura, and N. Nakashima, "Noncovalent porphyrin-functionalized single-walled carbon nanotubes in solution and the formation of porphyrin-nanotube nanocomposites," Chemical Physics Letters, vol. 378, no. 5-6, pp. 481485, 2003.

[6] H. Li, B. Zhou, Y. Lin et al., "Selective Interactions of porphyrins with semiconducting single-walled carbon nanotubes," Journal of the American Chemical Society, vol. 126, no. 4, pp. 1014-1015, 2004.

[7] D. M. Guldi, H. Taieb, G. M. A. Rahman, N. Tagmatarchis, and M. Prato, "Novel photoactive single-walled carbon nanotubeporphyrin polymer wraps: efficient and long-lived intracomplex charge separation," Advanced Materials, vol. 17, no. 7, pp. 871-875, 2005.

[8] J. Chen and C. P. Collier, "Noncovalent functionalization of single-walled carbon nanotubes with water-soluble porphyrins," Journal of Physical Chemistry. B, vol. 109, no. 16, pp. 7605-7609, 2005.

[9] H. Li, R. B. Martin, B. A. Harruff, R. A. Carino, L. F. Allard, and Y. P. Sun, "Single-walled carbon nanotubes tethered with porphyrins: synthesis and photophysical properties," Advanced Materials, vol. 16, no. 11, pp. 896-900, 2004.

[10] E. N. Mhuircheartaigh, S. Giordani, and W. J. Blau, "Linear and nonlinear optical characterization of a tetraphenylporphyrin — carbon nanotube composite system," Journal of Physical Chemistry. B, vol. 110, no. 46, pp. 23136-23141, 2006.

[11] J. Wang and W. J. Blau, "Inorganic and hybrid nanostructures for optical limiting," Journal of Optics. A, vol. 11, no. 2, Article ID 024001, 2009.

[12] G. X. Chen, M. H. Hong, L. S. Tan et al., "Optical limiting phenomena of carbon nanoparticles prepared by laser ablation in liquids," Journal of Physics: Conference Series, vol. 59, no. 1, pp. 289-292, 2007.

[13] W. W. Kalisch and M. O. Senge, "Synthesis and structural characterization of nonplanar tetraphenylporphyrins with graded degree of $\beta$-ethyl substitution," Tetrahedron Letters, vol. 37, no. 8, pp. 1183-1186, 1996.

[14] M. O. Senge and W. W. Kalisch, "Synthesis and structural characterization of nonplanar tetraphenylporphyrins and their metal complexes with graded degrees of $\beta$-ethyl substitution," Inorganic Chemistry, vol. 36, no. 26, pp. 6103-6116, 1997.

[15] L. D. Sparks, C. J. Medforth, M. S. Park et al., "Metal dependence of the nonplanar distortion of octaalkyltetraphenylporphyrins," Journal of the American Chemical Society, vol. 115, no. 2, pp. 581-592, 1993.

[16] J. E Seto, S. I. Tamura, N. Asai, N. Kishii, Y. Kijima, and N. Matsuzawa, "Macrocyclic functional dyes: applications to optical disk media, photochemical hole burning and nonlinear 
optics," Pure and Applied Chemistry, vol. 68, no. 7, pp. 14291434, 1996.

[17] M. Calvete, G. Y. Yang, and M. Hanack, "Porphyrins and phthalocyanines as materials for optical limiting," Synthetic Metals, vol. 141, no. 3, pp. 231-243, 2004.

[18] L. Vivien, E. Anglaret, D. Riehl et al., "Optical limiting properties of singlewall carbon nanotubes," Optics Communications, vol. 174, no. 1-4, pp. 271-275, 2000.

[19] S. R. Mishra, H. S. Rawat, S. C. Mehendale et al., "Optical limiting in single-walled carbon nanotube suspensions," Chemical Physics Letters, vol. 317, no. 3-5, pp. 510-514, 2000.

[20] J. E. Riggs, D. B. Walker, D. L. Carroll, and Y. P. Sun, "Optical limiting properties of suspended and solubilized carbon nanotubes," Journal of Physical Chemistry. B, vol. 104, no. 30, pp. 7071-7076, 2000.

[21] P. Nikolaev, M. J. Bronikowski, R. K. Bradley et al., "Gasphase catalytic growth of single-walled carbon nanotubes from carbon monoxide," Chemical Physics Letters, vol. 313, no. 1-2, pp. 91-97, 1999.

[22] M. Sheik-Bahae, A. A. Said, T. H. Wei, D. J. Hagan, and E. W. van Stryland, "Sensitive measurement of optical nonlinearities using a single beam," IEEE Journal of Quantum Electronics, vol. 26, no. 4, pp. 760-769, 1990.

[23] S. M. O'Flaherty, S. V. Hold, M. J. Cook et al., "Molecular engineering of peripherally and axially modified phthalocyanines for optical limiting and nonlinear optics," Advanced Materials, vol. 15, no. 1, pp. 19-32, 2003.

[24] H. Sun, "Compass: an ab initio force-field optimized for condensed-phase applications-overview with details on alkane and benzene compounds," Journal of Physical Chemistry. B, vol. 102, no. 38, pp. 7338-7364, 1998.

[25] E. N. Mhuircheartaigh, W. J. Blau, M. Prato, and S. Giordani, "Sonication of porphyrin-nanotube composites: a cautionary tale," Physica Status Solidi. B, vol. 244, no. 11, pp. 4227-4230, 2007.

[26] M. O. Senge, C. J. Medforth, L. D. Sparks, J. A. Shelnutt, and K. M. Smith, "A planar dodecasubstituted porphyrin," Inorganic Chemistry, vol. 32, no. 9, pp. 1716-1723, 1993.

[27] M. O. Senge, M. W. Renner, W. W. Kalisch, and J. Fajer, "Molecular structure of (5,10,15,20-tetrabutyl-2,3,7,8,12,13, 17,18-octaethylporphyrinato)nickel(II)- correlation of nonplanarity with frontier orbital shifts," Journal of the Chemical Society, Dalton Transactions, no. 3, pp. 381-385, 2000.

[28] N. C. Maiti and M. Ravikanth, "Effects of non-planarity and $\beta$ substitution on the singlet-excited-state properties of baskethandle porphyrins," Journal of the Chemical Society, Faraday Transactions, vol. 92, no. 7, pp. 1095-1100, 1996.

[29] A. Rosa, G. Ricciardi, E. J. Baerends, A. Romeo, and L. M. Scolaro, "Effects of porphyrin core saddling, meso-phenyl twisting, and counterions on the optical properties of mesotetraphenylporphyrin diacids: the $\left[\mathrm{H}_{4} \mathrm{TPP}\right](\mathrm{X})_{2}(\mathrm{X}=\mathrm{F}, \mathrm{Cl}, \mathrm{Br}$, I) series as a case study," Journal of Physical Chemistry. A, vol. 107, no. 51, pp. 11468-11482, 2003.

[30] D. Baskaran, J. W. Mays, X. P. Zhang, and M. S. Bratcher, "Carbon nanotubes with covalently linked porphyrin antennae: photoinduced electron transfer," Journal of the American Chemical Society, vol. 127, no. 19, pp. 6916-6917, 2005.

[31] A. Satake, Y. Miyajima, and Y. Kobuke, "Porphyrin-carbon nanotube composites formed by noncovalent polymer wrapping," Chemistry of Materials, vol. 17, no. 4, pp. 716-724, 2005.

[32] D. M. Guldi, H. Taieb, G. M. A. Rahman, N. Tagmatarchis, and M. Prato, "Novel photoactive single-walled carbon nanotube-porphyrin polymer wraps: efficient and long-lived intracomplex charge separation," Advanced Materials, vol. 17, no. 7, pp. 871-875, 2005.

[33] G. M. A. Rahman, D. M. Guldi, S. Campidelli, and M. Prato, "Electronically interacting single wall carbon nanotubeporphyrin nanohybrids," Journal of Materials Chemistry, vol. 16 , no. 1, pp. 62-65, 2006.

[34] J. N. Miller, Standards in Fluorescence Spectrometry, Chapter 5, Chapman \& Hall, New York, NY, USA, 1981.

[35] C. A. Parker and W. T. Rees, "Fluorescence spectrometry. A review," The Analyst, vol. 87, no. 1031, pp. 83-111, 1962.

[36] J. N. Coleman, A. Fleming, S. Maier et al., "Binding kinetics and SWNT bundle dissociation in low concentration polymernanotube dispersions," Journal of Physical Chemistry. B, vol. 108, no. 11, pp. 3446-3450, 2004.

[37] M. Sheik-Bahae, A. A. Said, D. J. Hagan, M. J. Soileau, and E. W. van Stryland, "Nonlinear refraction and optical limiting in thick media," Optical Engineering, vol. 30, pp. 1228-1235, 1991.

[38] E. Fazio, F. Neri, S. Patanè, L. D'Urso, and G. Compagnini, "Optical limiting effects in linear carbon chains," Carbon, vol. 49, no. 1, pp. 306-310, 2011.

[39] J. W. Perry, K. Mansour, I. Y. S. Lee et al., "Organic optical limiter with a strong nonlinear absorptive response," Science, vol. 273, no. 5281, pp. 1533-1536, 1996. 

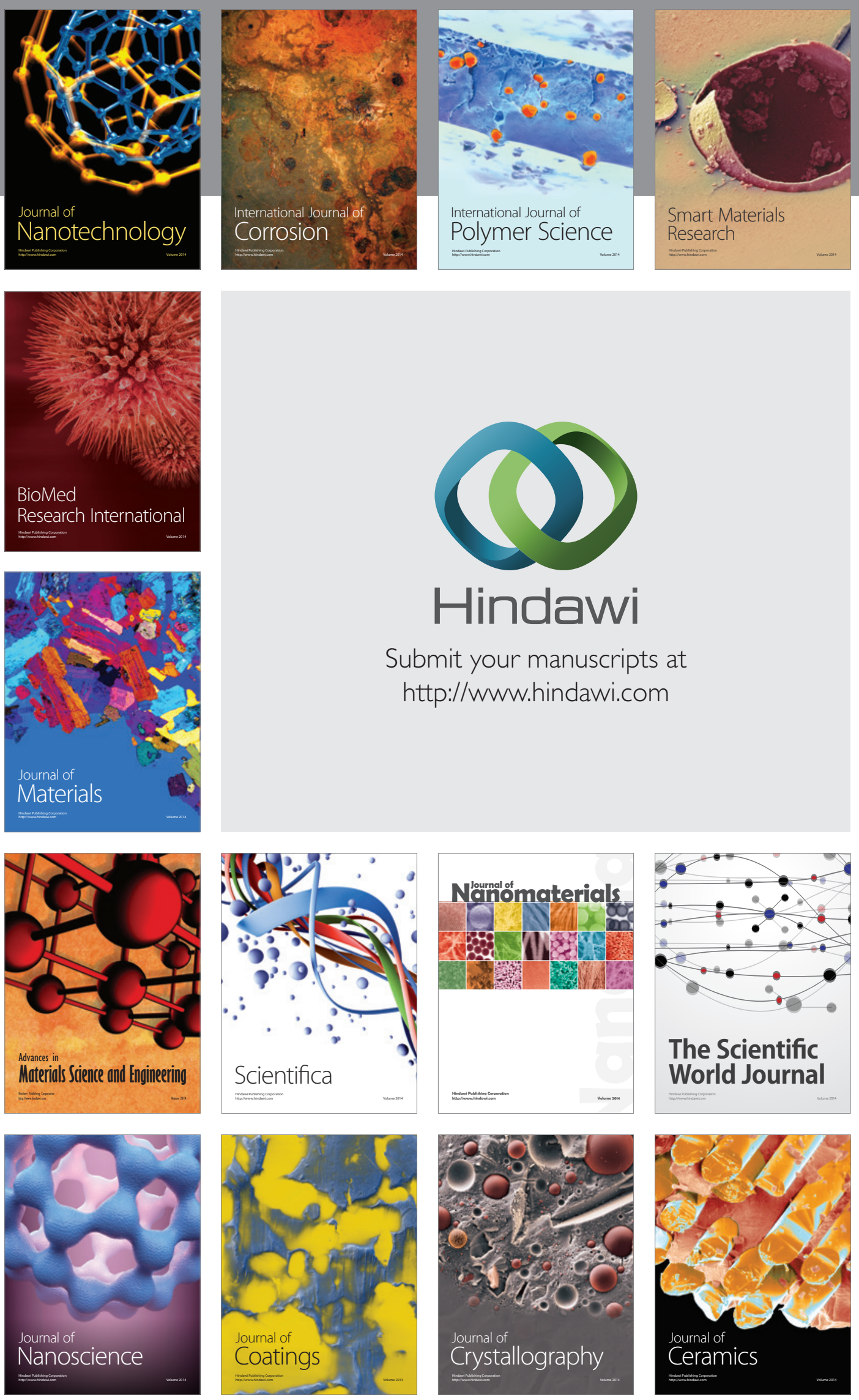

The Scientific World Journal

Submit your manuscripts at

http://www.hindawi.com

\section{World Journal}

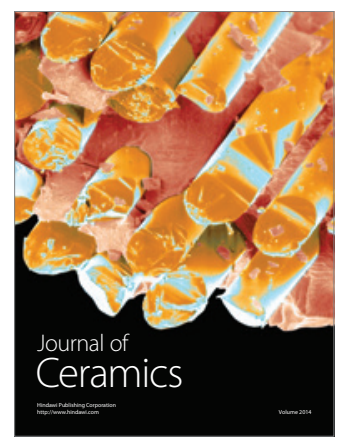

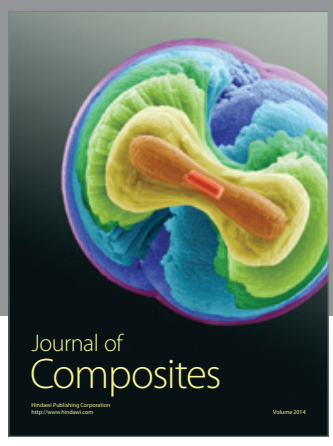
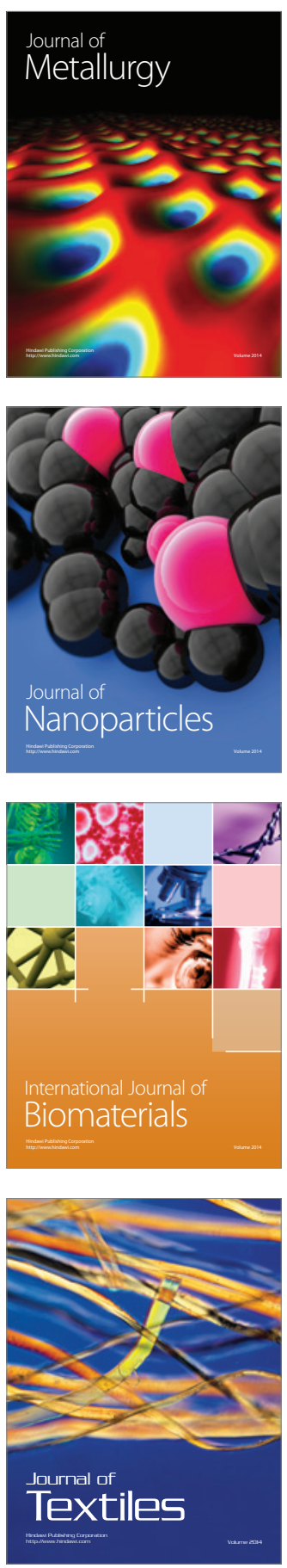